\title{
Intraoperative monopolar mapping during 5-ALA-guided resections of glioblastomas adjacent to motor eloquent areas: evaluation of resection rates and neurological outcome
}

\author{
Philippe Schucht, M.D., ${ }^{1}$ Kathleen Seidel, M.D., ${ }^{1}$ Jürgen Beck, M.D., ${ }^{1}$ \\ Michael Murek, M.D., ${ }^{1}$ Astrid Jilch, M.D., ${ }^{1}$ Roland Wiest, M.D., ${ }^{2}$ \\ Christian Fung, M.D., ${ }^{1}$ And Andreas Raabe, M.D. ${ }^{1}$ \\ Departments of ${ }^{I}$ Neurosurgery and ${ }^{2}$ Neuroradiology, Inselspital, Bern University Hospital, Bern, Switzerland
}

Object. Resection of glioblastoma adjacent to motor cortex or subcortical motor pathways carries a high risk of both incomplete resection and postoperative motor deficits. Although the strategy of maximum safe resection is widely accepted, the rates of complete resection of enhancing tumor (CRET) and the exact causes for motor deficits (mechanical vs vascular) are not always known. The authors report the results of their concept of combining monopolar mapping and 5-aminolevulinic acid (5-ALA)-guided surgery in patients with glioblastoma adjacent to eloquent tissue.

Methods. The authors prospectively studied 72 consecutive patients who underwent 5-ALA-guided surgery for a glioblastoma adjacent to the corticospinal tract (CST; $<10 \mathrm{~mm}$ ) with continuous dynamic monopolar motor mapping (short-train interstimulus interval $4.0 \mathrm{msec}$, pulse duration $500 \mu \mathrm{sec}$ ) coupled to an acoustic motor evoked potential (MEP) alarm. The extent of resection was determined based on early ( $<48$ hours) postoperative MRI findings. Motor function was assessed 1 day after surgery, at discharge, and at 3 months.

Results. Five patients were excluded because of nonadherence to protocol; thus, 67 patients were evaluated. The lowest motor threshold reached during individual surgery was as follows (motor threshold, number of patients): $>20 \mathrm{~mA}, \mathrm{n}=8 ; 11-20 \mathrm{~mA}, \mathrm{n}=13 ; 6-10 \mathrm{~mA}, \mathrm{n}=10 ; 4-5 \mathrm{~mA}, \mathrm{n}=13$; and 1-3 mA, $\mathrm{n}=23$. Motor deterioration at postsurgical Day 1 and at discharge occurred in $30 \%(\mathrm{n}=20)$ and $10 \%(\mathrm{n}=7)$ of patients, respectively. At 3 months, 3 patients $(4 \%)$ had a persisting postoperative motor deficit, 2 caused by vascular injury and 1 by mechanical injury. The rates of intra- and postoperative seizures were $1 \%$ and $0 \%$, respectively. Complete resection of enhancing tumor was achieved in $73 \%$ of patients $(49 / 67)$ despite proximity to the CST.

Conclusions. A rather high rate of CRET can be achieved in glioblastomas in motor eloquent areas via a combination of 5-ALA for tumor identification and intraoperative mapping for distinguishing between presumed and actual motor eloquent tissues. Continuous dynamic mapping was found to be a very ergonomic technique that localizes the motor tissue early and reliably.

(http://thejns.org/doi/abs/10.3171/2014.10.FOCUS14524)

\section{KeY Words - corticospinal tract $\quad$ glioblastoma $\quad$ c tumor surgery $\bullet$ intraoperative neuromonitoring • motor evoked potential • subcortical mapping}

A GROWING body of evidence indicates that surgery prolongs survival in glioblastoma patients if an extensive part of the contrast-enhancing tumor is resected, ${ }^{21,34,49}$ although all therapies for glioblastoma are essentially palliative for most patients. ${ }^{51}$ The greatest survival benefit is observed in patients with complete resection of enhancing tumor (CRET). ${ }^{41}$ However, removal of

\footnotetext{
Abbreviations used in this paper: CRET = complete resection of enhancing tumor; $\mathrm{CST}=$ corticospinal tract; CUSA = Cavitron Ultrasonic Surgical Aspirator; DCS = direct cortical stimulation; EEG = electroencephalography; GTR = gross-total resection; KPS = Karnofsky Performance Scale; MEP = motor evoked potential; MRC $=$ Medical Research Council; MT = motor threshold; SSEP = somatosensory evoked potential; STR $=$ subtotal resection; TES $=$ transcranial electrical stimulation; $\mathrm{TOF}=$ train of five; 5 -ALA $=$ 5-aminolevulinic acid.
}

the final $1 \%-2 \%$ of the contrast-enhancing tumor carries not only the greatest impact from an oncological point of view ${ }^{20,42,44,45}$ but also the greatest risk for neurological impairment, especially in glioblastomas adjacent to motor eloquent areas. Several newly developed technical procedures, such as the use of 5-aminolevulinic acid $(5-\mathrm{ALA})^{41}$ and intraoperative MRI, ${ }^{39}$ have been proven to increase the rate of CRET, leading resections deeper into the white matter, which in turn endangers neurological function. Balancing the oncological benefit of more extensive resections and the prerequisite of sparing neurological functionality (maximum safe resection) is particularly challenging in patients with glioblastoma close to the corticospinal tract (CST).

Electrical stimulation is a validated intraoperative technique for identifying motor eloquent areas. ${ }^{1,3,29}$ Inter- 
mittent subcortical mapping $2,12,14,19,33,47$ or continuous dynamic mapping ${ }^{31}$ are used intraoperatively to localize the CST and thereby reduce the risk of motor deficits.

Although the strategy of maximum safe resection is widely accepted, the rates of CRET and the exact causes of motor deficits (mechanical vs vascular) despite the use of electrophysiological techniques are not well known. In this prospective study on glioblastoma surgery, we evaluated our previously described mapping technique that allows continuous subcortical stimulation in the surgical cavity during resection. Because continuous stimulation is synchronized with tumor removal, we can overcome the temporal and spatial limitations of classic subcortical mapping of the CST. ${ }^{31}$ This study investigated the combined use of continuous mapping and 5-ALA imaging during surgery of glioblastomas adjacent to motor eloquent areas. Outcome was based on postoperative neurological deficits and the extent of tumor resection.

\section{Methods}

\section{Study Design and Patient Population}

We conducted a prospective study on 72 patients who underwent surgery in our Department of Neurosurgery between August 1, 2010, and July 31, 2014, for a glioblastoma adjacent to the CST. Localization of the CST was determined on preoperative MRI based on imaging criteria for the primary motor cortex and diffusion tensor imaging (performed in 61 of 72 patients). Five patients were excluded from analysis due to nonadherence to protocol; 4 did not receive 5-ALA and 1 did not undergo postoperative MRI. The diagnosis was confirmed by histopathology in all cases. Twenty-six of these patients were previously included in a proof-of-concept analysis. ${ }^{31}$ The mean patient age was 56 years (range 22-77 years). All patients signed informed consent for the surgery and the prospective data collection. The analysis was approved by the local institutional ethics review board (Cantonal Ethic Commission [Kantonale Ethikkommission], Bern University Hospital, Bern, Switzerland).

We used the Medical Research Council (MRC) scale $^{24}$ (Grades M1-M5) for motor function analysis and the Karnofsky Performance Scale (KPS) ${ }^{17}$ for general performance analysis, both pre- and postoperatively. The median preoperative KPS score was 80 (range 50-100). Twenty-two patients had preoperative motor impairment; the MRC grade was M4 in 18 patients $(27 \%)$, M3 in 3 (4\%), and M2 in 1 (1\%). Forty-five patients $(67 \%)$ had intact motor function before surgery. Neurological deficits that had resolved by the time of the 3-month visit were classified as temporary. Any persisting deficit was classified as permanent.

\section{Intraoperative Electrophysiology}

General anesthesia was used for all surgeries. Anesthesia was induced using propofol (1-2 $\mathrm{mg} / \mathrm{kg}$ body weight), fentanyl (1-2 $\mu \mathrm{g} / \mathrm{kg}$ body weight), and remifentanil (1-2 $\mu \mathrm{g} / \mathrm{kg}$ body weight) and was maintained with propofol (100-200 $\mu \mathrm{g} / \mathrm{kg} / \mathrm{min})$ and remifentanil (0.5 $\mu \mathrm{g} /$ $\mathrm{kg} / \mathrm{hr}$ ). A short-acting relaxant (rocuronium bromide [Es- meron] $0.6 \mathrm{mg} / \mathrm{kg}$ body weight) was administered for intubation only. The "train-of-four" technique was used to test recovery from muscle relaxation. ${ }^{48}$

We used the ISIS system (Inomed Co.) equipped with a constant current stimulator (OSIRIS, maximal stimulator output of $220 \mathrm{~mA}$, Inomed Co.) for intraoperative neurophysiological monitoring and mapping. Motor evoked potentials (MEPs) of the contralateral target muscles (facial as well as distal and proximal upper and lower limbs) were recorded using pairs of needle electrodes. Details about the direct cortical stimulation (DCS)-MEP monitoring using a strip electrode have previously been published. ${ }^{38}$ Transcranial electrical stimulation (TES) was performed if the placement of a grid electrode was impossible. For TES we used the same stimulation parameters as for DCS and placed the corkscrew electrodes at $\mathrm{C} 1, \mathrm{C} 2, \mathrm{C} 3, \mathrm{C} 4$, and $\mathrm{Cz}$ according to the 10-20 electroencephalography (EEG) system.8,46 Somatosensory evoked potential (SSEP) corkscrew scalp electrodes were used for seizure monitoring. Different derivations of recordings were applied simultaneously, e.g., C3-Fz, C4$\mathrm{Fz}, \mathrm{C} 3-\mathrm{C} 4, \mathrm{C} 3-\mathrm{Cz}, \mathrm{C} 4-\mathrm{Cz}$. Additionally, SSEPs of all 4 extremities were monitored. Localization of the central sulcus was done with the help of the median nerve phasereversal technique.

\section{Concept of Continuous Dynamic Mapping}

Continuous dynamic mapping is achieved using a suction device with monopolar mapping capabilities (Fig. 1). "Dynamic" refers to the quickly changing location of the suction tip, which enables mapping at the current site of tissue removal. Stimulation $(2 \mathrm{~Hz})$ is activated by connecting the cable for the standard monopolar fingerstick probe directly to the suction device (Fig. 1). The surface of the suction probe is isolated to limit electrical contact to the tip of the device. Otherwise, identical stimulation parameters as for the classic monopolar train-of-five (TOF; interstimulus interval $4.0 \mathrm{msec}$, pulse duration $500 \mu \mathrm{sec}$ ) were used. We previously demonstrated a 1:1 correlation

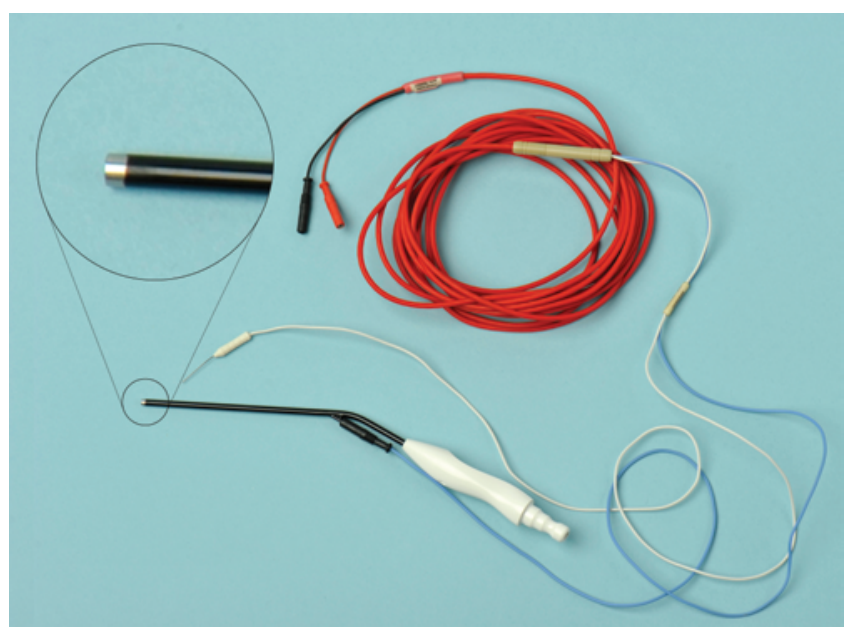

FIG. 1. Continuous dynamic mapping device. The suction tip includes monopolar mapping capabilities. Continuous stimulation current can be delivered via the connector beneath the handle. The device is electrically isolated up to its tip. 


\section{Continuous dynamic mapping for glioblastoma near eloquent areas}

between motor mapping thresholds measured by the classic fingerstick probe and our suction-stimulator device. ${ }^{31}$

We use a continuous dynamic mapping device like a standard suction device to clear the resection site of blood, irrigation fluid, and debris at the exact location where a resection instrument such as the Cavitron Ultrasonic Surgical Aspirator (CUSA) or bipolar forceps is deployed for tumor removal by the other hand. Thus, the tip of the mapping suction device delivers the stimulation current directly at the location where the CUSA or bipolar forceps destroys and removes tumor tissue, thereby providing dynamic mapping at changing locations. The 2-Hz frequency ensures that a reliable feedback consisting of 3 consecutive MEPs is obtained within a contact time of 1.5 seconds. Ultimately, we use the continuous dynamic mapping device as a "CST radar," i.e., to test whether an MEP can be elicited with a chosen stimulation intensity around the current site of resection while keeping in mind the 1:1 distance-to-current relationship. A high-pitch sound is delivered with every single train of stimulation to inform the surgeon that adequate current was delivered to the tissue while the continuous dynamic device was in use (acoustic negative control). A low-pitch alert sound, easy to differentiate even in a noisy environment, is only delivered if the amplitude of an MEP response in the recorded muscles reaches the threshold of $30 \mu \mathrm{V}$. MEP responses can also be observed in both freerunning and triggered electromyography screens.

\section{Intraoperative Handling of the Continuous Dynamic Mapping Device}

The continuous dynamic mapping device is used when the surgeon is within $10-15 \mathrm{~mm}$ of the CST, as determined by neuronavigation or according to anatomical orientation of the surgeon (Fig. 2). We start with an initial stimulation intensity of 10-15 mA, which corresponds to about $10-15 \mathrm{~mm}$ from the CST. ${ }^{16,27,28,30,38}$ The instant an MEP is elicited, the surgeon is immediately alerted by the change of the high-pitch negative control sound to a low-pitch alert sound. The stimulation intensity is then reduced in 2-mA steps, allowing the surgeon to continue tumor removal until another low-pitch alert sound indicates that resection is getting closer to the CST. Lowering of the stimulation intensity corresponds to a reduction of the remaining distance to the CST and is reflected in the term "quantitative mapping." 31,38 Resection was usually continued until a motor threshold (MT) of $3 \mathrm{~mA}$ was reached. Surgery beyond an MT of $3 \mathrm{~mA}$ was only performed in select cases when the surgeon believed that a CRET could be achieved, and provided that DCS-MEPs remained stable. In all cases the surgeon stopped if 1- or 2-mA stimulation intensity triggered an MEP indicating very close proximity to the CST. Resection was immediately paused in cases of DCS-MEP alterations. To avoid permanent postoperative motor deficits, we stopped removal of tumor tissue if DCS-MEP alterations persisted after a 15-minute pause.

\section{5-ALA}

Patients received 5-ALA (20 mg/kg body weight,
Medac) 3 hours before the induction of anesthesia (range 2-4 hours). Surgery was performed using a neurosurgical microscope (Zeiss) with a fluorescence kit that enabled repetitive switching from xenon illumination to violetblue excitation light. 5-ALA-induced fluorescence was used repeatedly during tumor tissue resection, especially at the resection borders. Resection was continued with the CUSA device under the blue light until 1) surgery was stopped due to proximity to the CST as indicated by the continuous dynamic mapping device or 2) all red and pink fluorescent tissue was removed. At the end of surgery, the resection cavity was again checked with the blue light, and the completeness of 5-ALA resection was noted.

\section{Imaging and Extent of Resection}

Patients were required to undergo preoperative MRI (T1-weighted with and without gadolinium enhancement as well as T2-weighted imaging) no longer than 14 days before surgery and protocol-specified postoperative MRI within 48 hours of surgery. MRI was performed using a 1.5- or a 3-T scanner with a head coil. Unenhanced and enhanced (magnetization-prepared rapid-acquisition gradient echocardiography sequences with $0.1 \mathrm{mmol} / \mathrm{kg}$ body weight gadolinium-DTPA given intravenously) T1 sequences without gap were obtained $(256 \times 256$ matrix, rectangular field of view, 1-mm slice thickness). Contrast enhancement within $10 \mathrm{~mm}$ of the CST was required as a study inclusion criterion. The CST was localized after fiber tracking using diffusion tensor imaging based on preoperative MRI. The extent of resection was determined by the senior neuroradiologist (R.W.); CRET was declared in the absence of any tumor-suspicious contrast enhancement on postoperative MRI, ${ }^{50}$ and all resections were considered subtotal resections (STRs). We used iPlan 3.0.2 software (VectorVision, Brainlab) to determine the exact volume of the contrast-enhancing tumor on pre- and postoperative MRI. Our monopolar mapping approach was designed to minimize mechanical injury to the CST. To analyze the ability of this approach to limit the rate of mechanical injury, a distinction between mechanical (direct injury) and microvascular injury (indirect ischemic changes after injury of microvasculature) is helpful. Mechanical and microvascular injuries (ischemia) to the CST were defined as a partial discontinuation of the CST on FLAIR sequences and diffusion-weighted imaging restrictions, respectively.

\section{Results}

Motor Thresholds With Continuous Dynamic Quantitative Mapping

All procedures were technically successful. Lowest individual MTs were as follows (MT, number of patients): $>20 \mathrm{~mA}, \mathrm{n}=8 ; 11-20 \mathrm{~mA}, \mathrm{n}=13 ; 6-10 \mathrm{~mA}, \mathrm{n}=10 ; 4-5$ $\mathrm{mA}, \mathrm{n}=13$; and 1-3 mA, $\mathrm{n}=23$. MEP monitoring (DCS, TES, or combined method) showed stable signals in 48 patients, unspecific changes in 14, and sudden or irreversible alterations in 5. No case showed a loss of MEPs. 

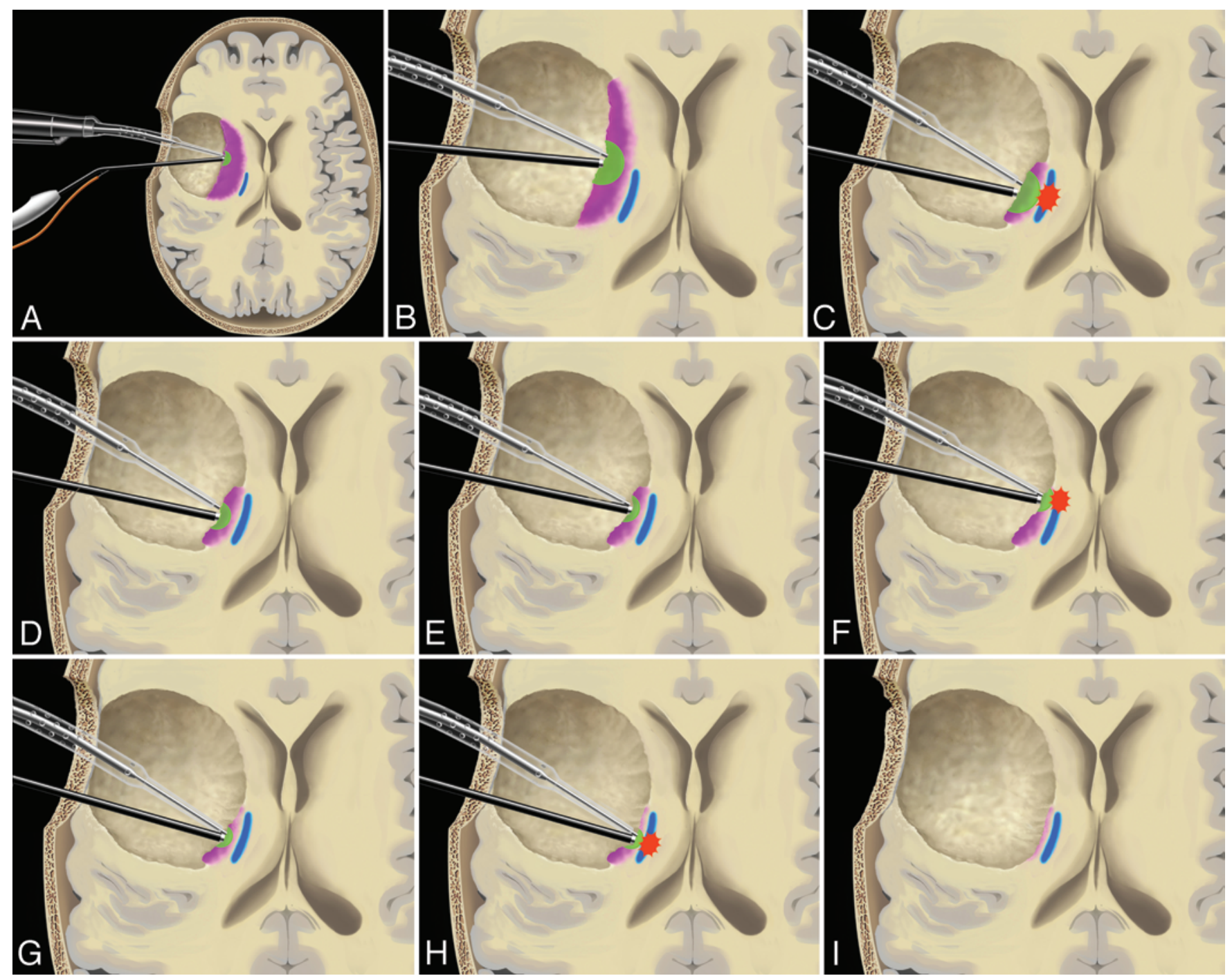

FIG. 2. Use of the continuous dynamic mapping device. The continuous dynamic mapping device is used within $10 \mathrm{~mm}$ of the CST (A). Resection is continued with an initial stimulation intensity of 10-15 mA (corresponding to a distance of 10-15 mm from the CST; B). The instant an MEP is elicited (C), the surgeon is alerted by the change of the high-pitch negative control sound to a low-pitch alert sound. The stimulation intensity is then reduced by $2 \mathrm{~mA}$, and the resection is continued at sites negative for the set current ( $D$ and $E$ ), until again the acoustic switch from the negative control to the alert sound warns the surgeon that the MT has been reached (F). This stepwise approach is continued until a minimal threshold is reached (G-I). Pink indicates tumor tissue, blue indicates CST fibers, green indicates a region of active resection, and red indicates that an alert sound has occurred. Copyright Andreas Raabe. Published with permission.

\section{5-ALA Fluorescence, Reasons to Abort Further Removal and Extent of Resection}

5-ALA fluorescence was useful for tumor tissue identification in all 67 cases. Thirty-eight patients (57\%) had a 5-ALA complete resection. Surgery was stopped in 16 patients based on mapping findings (mean MT 3 $\mathrm{mA}$, range $1-5 \mathrm{~mA}$; stable MEP signals in 14 patients and unspecific MEP alterations in 2 patients) despite persisting 5-ALA fluorescence. According to postoperative MRI, however, CRET was achieved in 9 of these 16 cases. Overall, 49 patients had CRET (73\%) (Fig. 3). Eighteen patients $(27 \%)$ had an STR with a median remnant of $0.93 \mathrm{~cm}^{3}$ (interquartile range $0.268-2.026$ ). In these patients with STR, the reasons for STR were: 1) infiltration over the midline (3 patients); 2) infiltration in eloquent but nonmotor areas (determined according to anatomical features on preoperative MRI; optic radiation and frontal speech areas in 1 patient each and basal ganglia in 2 patients); 3) tumor resection aborted after a safe complete resection appeared impossible based on mapping (6 patients, at a mean MT of $3 \mathrm{~mA}$ ); 4) irreversible changes of MEP (2 patients); and 5) unknown (3 patients).

\section{Postoperative Motor Performance}

Postoperative worsening in motor status on the 1st day after surgery was observed in 20 patients (30\%). At discharge, 13 patients $(19 \%)$ had complete motor recovery and 7 patients (10\%) had persisting deficits. At the 


\section{Continuous dynamic mapping for glioblastoma near eloquent areas}

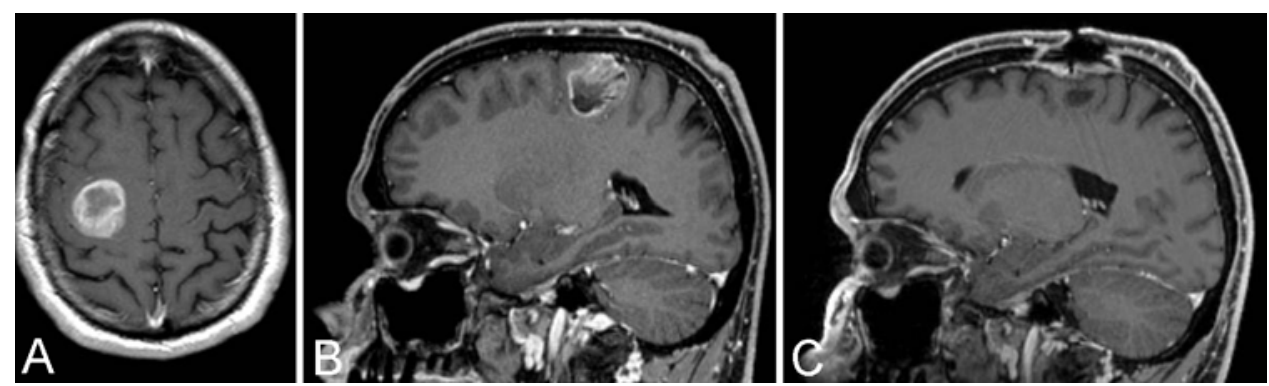

FIG. 3. Pre- and postoperative contrast-enhanced MR images. Preoperative axial (A) and sagittal (B) images revealed a lesion in the right precentral gyrus. Postoperative imaging showed CRET (C). Resection was stopped at a lowest MT of $1 \mathrm{~mA}$ despite persisting 5-ALA fluorescence.

3-month visit, 3 patients (4\%) presented with a persisting motor deficit. One patient sustained an injury to the $\mathrm{A}_{2}$ segment of the anterior cerebral artery (lowest mapping MT $20 \mathrm{~mA}$; sudden, irreversible threshold increase $>4 \mathrm{~mA}$ in DCS-MEP stimulation). The patient did not recover from a relative worsening of motor status (from Grade M5 to M3). Two patients had sudden, irreversible threshold increase ( $>4 \mathrm{~mA}$ in DCS-MEP stimulation intensity; lowest mapping MT $4 \mathrm{~mA}$ ) and had permanent motor worsening (from Grade M5 to M4). Postoperative MRI revealed mechanical damage in the internal capsule in 1 patient and ischemic changes (revealed as diffusionweighted imaging restrictions) in the precentral gyrus in 2 others. Postoperative motor worsening was due to a supplementary motor area syndrome in 3 patients; all had full recovery within 3 months. The median KPS score for all patients at 3 months was 80, ranging from 40 to 100 .

\section{Seizures}

Continuous EEG was performed per protocol in all patients during mapping. EEG depicted seizure activity during mapping in a single patient, where seizure activity was terminated by application of cold Ringers solution and intravenous bolus injection of clonazepam, without further consequences. No patient had seizures postoperatively until discharge. Thus, the rates of intra- and postoperative seizures were $1 \%$ and $0 \%$, respectively.

\section{Discussion}

Infiltration of presumed motor eloquent area based on preoperative MR images is a risk factor for both incomplete resection and postoperative motor deficit. . $^{1,6,23,31}$ Incomplete resection, on the other hand, is a risk factor for decreased survival. ${ }^{42}$ Hence, areas of presumed motor eloquence should be tested by intraoperative neuromonitoring to optimize the oncological benefit of surgery. ${ }^{10}$

Motor mapping is used to localize the CST and to determine the safe distance from the CST.,2,12 Gliomas in areas thought to be motor eloquent based on preoperative imaging have often proven to be resectable without permanent deficits after the area in question tested negative for motor function via intraoperative mapping. 1,2,4,6,9,11,13,26,35,38 Furthermore, a proof-of-concept analysis showed that tumors adjacent to motor eloquent areas may be safely removed down to MTs as low as $1-3 \mathrm{~mA}$, so long as MEP monitoring remains stable. ${ }^{31,37}$
An MT-to-distance relationship exists for subcortical monopolar TOF mapping of the CST.16,19,27 As a rule of thumb, $1 \mathrm{~mA}$ of current corresponds to $1 \mathrm{~mm}$ remaining distance to the CST. ${ }^{27,28}$ We previously demonstrated a safe window between high and low MTs for mechanical injury to the CST and showed that significant signal changes in MEP monitoring and permanent motor deficits do not occur above very low MTs of less than 1-2 mA. ${ }^{38}$ These data also support the hypothesis that insufficient spatial and temporal coverage of the surgical site due to use of an interruptive and punctiform mapping technique may contribute to motor deficits despite higher and apparently safe MTs (3-6 mA).

\section{How Close to the CST Can We Safely Resect Tumor Tissue?}

A safe window has been described for monopolar high-frequency TOF mapping between $20 \mathrm{~mA}$ and 3-5 $\mathrm{mA}^{16,19,25,27,28,47}$ or even 1-3 $\mathrm{mA}^{38}$ An MT within this range excludes mechanical damage of the CST and permanent motor deficit, provided that the measured MT is indeed the lowest in the resection cavity, the surgeon does not continue resection after mapping, and no vascular injury has occurred.

Recent data have suggested that the critically low mapping MTs associated with motor deficit are probably lower than previously thought. ${ }^{38}$ In cases in which there was a very low mapping MT of $1 \mathrm{~mA}, 75 \%$ of patients showed stable DCS-MEP or only unspecific reversible changes, and none of them had a permanent motor worsening at 3 months. However, the same study found nonvascular motor deficits occurring at MTs of 6,3, and $1 \mathrm{~mA}$, presumably because mapping was not performed continuously. ${ }^{38}$ The proof-of-concept analysis on continuous dynamic mapping confirmed the safety of low-threshold motor mapping without any permanent motor deficit due to mechanical injuries and a $3 \%$ rate of permanent motor deficits (2 patients) due to vascular injuries. ${ }^{31}$ Our rate of permanent motor deficit in glioblastoma adjacent to motor eloquent areas (3\% vascular injury and $1 \%$ mechanical injury; 2 and 1 patients, respectively) is in line with earlier results. In 1 patient injury to a branch of the anterior cerebral artery led to immediate and irreversible MT increment; this patient suffered a permanent motor deficit (Grade M3). Another patient had a sudden, irreversible MT increment during resection at a lowest MT of $4 \mathrm{~mA}$; postoperative MRI revealed a microvascular injury to the precentral gyrus and the patient sustained a permanent motor worsening from 


\section{P. Schucht et al.}

Grade M5 to M4. High rates of microvascular lesions have previously been reported in glioma surgery. ${ }^{15}$ Microvascular injuries may be of particular importance in glioblastoma due to tumor angiogenesis and recruitment of peritumoral vessels. Although our rate of neurological deficits due to microvascular injuries is low (3\%), we believe that the altered vascular structure in the tumors' periphery warrants special consideration. With respect to the relatively rare event of a mechanical injury, and in line with previous reports ${ }^{31}$ continuous mapping appears to provide a high degree of safety from mechanical injury (Fig. 3).

\section{The Need for Refinement of Mapping Technique}

Cumulating evidence indicates an exponential survival benefit after increasing the resection area of contrast-enhancing tissue, with the highest survival benefit seen after CRET. ${ }^{21,34,43}$ Similarly, post hoc analysis of the 5-ALA study ${ }^{41}$ showed a 4.9-month survival difference between patients who underwent gross-total resection (GTR) and those who underwent STR, even though the difference in residual tumor volume was marginal (0 vs $0.7 \mathrm{~cm}^{3}$ for GTR and STR, respectively). ${ }^{42}$ Delivering an optimal oncological result in patients with tumors adjacent to motor eloquent areas thus requires the ability to resect tumor tissue down to very low MTs (1-2 mA). Optimally, the topographical location of the CST relative to the site of resection should be known continuously to prevent inadvertent injury to motor fibers, especially when working close to the CST. Continuous (temporal coverage) and dynamic (spatial coverage) mapping can be achieved by integrating the mapping probe at the tip of a suction device with the stimulating tip, i.e., the mapping probe is in contact with the tissue at the site of resection. ${ }^{31}$ With use of continuous dynamic mapping, the surgeon starts with a stimulation intensity of about 10-15 mA after image guidance indicates a distance of about 10-15 $\mathrm{mm}$ from the CST. A key principle is to perform resection only at MEP-negative sites. With this technique, the tumor is always removed first at areas farther away from the CST. If there are tumor remnants at MEP-positive sites (indicated acoustically by an MEP-triggered switch from the negative control sound to the alert sound), the stimulation intensity is decreased by $2 \mathrm{~mA}$ and resection can be resumed (Fig. 2). Working on MEP-positive sites risks resecting into areas in which the MT is actually lower than the applied stimulation intensity, and motor deficits may occur even though stimulation intensity was applied at an apparently safe distance. We generally stop further resection at an MT of $3 \mathrm{~mA}$ if an STR was planned based on preoperative MRI findings. Resections beyond $3 \mathrm{~mA}$ are only performed if achieving CRET appears feasible and are stopped in all cases once an MEP-positive response is found at $1 \mathrm{~mA}$, regardless of DCS-MEP stability.

\section{The Benefit of Combining 5-ALA and Mapping}

The ability of 5-ALA to reveal solid and coalescent tumor and thus to facilitate GTR/CRET has previously been demonstrated. ${ }^{41}$ Use of 5-ALA was associated with a higher rate of temporary (but not permanent) neurological deficits, suggesting a higher invasiveness of 5-ALAguided surgery compared with white light surgery. ${ }^{41,42}$
5-ALA has a higher sensitivity for detecting infiltrating tumor than gadolinium-enhanced T1-weighted MRI.,40 The usefulness of a higher sensitivity was demonstrated in a volumetric analysis of 5-ALA-guided surgeries, which revealed that the volume of 5-ALA fluorescent tissue is significantly larger than contrast enhancement on preoperative MRI. 5-ALA fluorescence surpassed gadolinium enhancement by up to $10 \mathrm{~mm}$, potentially endangering nearby eloquent structures such as the CST. ${ }^{36}$ Although a significant correlation between the degree of infiltration and the intensity of fluorescence has been reported, areas of relatively little infiltration, or even those without infiltration, may show fluorescence of various intensities. ${ }^{32}$ 5-ALA is therefore inadequate as a stand-alone method for resections adjacent to eloquent areas. In the current study, resection was stopped in 16 patients due to low MTs (mean MT $3 \mathrm{~mA}$ ) despite persisting 5-ALA fluorescence. However, 9 of these 16 patients had a CRET according to postoperative MRI findings, emphasizing the increased sensitivity of 5-ALA compared with gadolinium. This finding underlines the complementary benefit of both methods; while 5-ALA fluorescence is helpful in optimizing the oncological benefit of surgery, continuous dynamic mapping shows how far surgery may go without compromising neurological outcome.

\section{Comparison of Motor Deficit Rates to the Literature}

Our motor deficit rate of $4 \%$ is in line with previous results with low-threshold $(<3-5 \mathrm{~mA})$ subcortical motor mapping and with our proof-of-concept analysis of continuous dynamic mapping. ${ }^{31,38}$ Our $4 \%$ rate of permanent motor deficit compares favorably with the literature, where deficit rates between $3.5 \%$ and $17 \%$ are reported. . $18,22,27,30,52^{-17}$ Of course, comparisons with published series are problematic due to patient selection issues, differing time points of outcome assessment, and various definitions of eloquence. However, the fact that an MT of $10 \mathrm{~mA}$ or less was reached in $70 \%$ of cases underlines the proximity to the CST of this series of tumors. Furthermore, the fact that only 1 patient had a mechanical injury even though resection was performed at or below the "critical" threshold of $5 \mathrm{~mA}$ in 36 of 67 patients (54\% of cases) emphasizes the safety of continuous dynamic mapping.

\section{Conclusions}

A rather high rate of CRET can be safely achieved even in glioblastomas in motor eloquent areas when using 5-ALA and mapping in combination. Our findings highlight the complementary benefit of 5-ALA and mapping; while 5-ALA fluorescence shows how far we should go to optimize the oncological benefit of surgery, continuous dynamic mapping shows how far resection may go without compromising neurological outcome. We believe that continuous dynamic mapping with acoustic feedback is a very ergonomic technique that allows the surgeon to easily and reliably localize the eloquent motor tissue. We also found that the volume of residual tumors remains very small when stopping at a threshold of $3 \mathrm{~mA}$. All but one of our patients had resections of more than $95 \%$ of tumor volume. 


\section{Continuous dynamic mapping for glioblastoma near eloquent areas}

\section{Acknowledgment}

We thank Alain Blank for the artwork in Fig. 2.

\section{Disclosure}

Dr. Schucht is a member of the MSD advisory board, and Dr. Raabe is a patent holder with Inomed.

Author contributions to this study and manuscript preparation include the following. Conception and design: Schucht, Raabe, Beck, Seidel. Acquisition of data: Schucht, Seidel, Beck, Murek, Jilch, Wiest, Fung. Analysis and interpretation of data: all authors. Drafting the article: Schucht. Critically revising the article: Schucht, Beck, Raabe, Seidel. Reviewed submitted version of manuscript: all authors. Approved the final version of the manuscript on behalf of all authors: Schucht. Statistical analysis: Schucht, Murek. Administrative/technical/material support: Raabe. Study supervision: Raabe.

\section{References}

1. Bello L, Castellano A, Fava E, Casaceli G, Riva M, Scotti G, et al: Intraoperative use of diffusion tensor imaging fiber tractography and subcortical mapping for resection of gliomas: technical considerations. Neurosurg Focus 28(2):E6, 2010

2. Berger MS, Hadjipanayis CG: Surgery of intrinsic cerebral tumors. Neurosurgery 61 (1 Suppl):279-305, 2007

3. Berger MS, Kincaid J, Ojemann GA, Lettich E: Brain mapping techniques to maximize resection, safety, and seizure control in children with brain tumors. Neurosurgery 25:786792, 1989

4. Berger MS, Rostomily RC: Low grade gliomas: functional mapping resection strategies, extent of resection, and outcome. J Neurooncol 34:85-101, 1997

5. Carrabba G, Fava E, Giussani C, Acerbi F, Portaluri F, Songa $\mathrm{V}$, et al: Cortical and subcortical motor mapping in rolandic and perirolandic glioma surgery: impact on postoperative morbidity and extent of resection. J Neurosurg Sci 51:45-51, 2007

6. Chang EF, Clark A, Smith JS, Polley MY, Chang SM, Barbaro NM, et al: Functional mapping-guided resection of low-grade gliomas in eloquent areas of the brain: improvement of longterm survival. Clinical article. J Neurosurg 114:566-573, 2011

7. Coburger J, Engelke J, Scheuerle A, Thal DR, Hlavac M, Wirtz CR, et al: Tumor detection with 5-aminolevulinic acid fluorescence and Gd-DTPA-enhanced intraoperative MRI at the border of contrast-enhancing lesions: a prospective study based on histopathological assessment. Neurosurg Focus 36(2): $\mathrm{E} 3,2014$

8. Deletis V: Intraoperative monitoring of the functional integrity of the motor pathways. Adv Neurol 63:201-214, 1993

9. Deletis V, Camargo AB: Transcranial electrical motor evoked potential monitoring for brain tumor resection. Neurosurgery 49:1488-1489, 2001

10. De Witt Hamer PC, Robles SG, Zwinderman AH, Duffau H, Berger MS: Impact of intraoperative stimulation brain mapping on glioma surgery outcome: a meta-analysis. J Clin Oncol 30:2559-2565, 2012

11. Duffau H: A personal consecutive series of surgically treated 51 cases of insular WHO Grade II glioma: advances and limitations. Clinical article. J Neurosurg 110:696-708, 2009 (Erratum in J Neurosurg 114:1486, 2011)

12. Duffau H, Capelle L, Denvil D, Sichez N, Gatignol P, Taillandier L, et al: Usefulness of intraoperative electrical subcortical mapping during surgery for low-grade gliomas located within eloquent brain regions: functional results in a consecutive series of 103 patients. J Neurosurg 98:764-778, 2003

13. Duffau H, Lopes M, Arthuis F, Bitar A, Sichez JP, Van Effenterre R, et al: Contribution of intraoperative electrical stimulations in surgery of low grade gliomas: a comparative study between two series without (1985-96) and with (1996-2003) functional mapping in the same institution. J Neurol Neurosurg Psychiatry 76:845-851, 2005

14. Fukaya C, Sumi K, Otaka T, Shijo K, Nagaoaka T, Kobayashi $\mathrm{K}$, et al: Corticospinal descending direct wave elicited by subcortical stimulation. J Clin Neurophysiol 28:297-301, 2011

15. Gempt J, Krieg SM, Hüttinger S, Buchmann N, Ryang YM, Shiban E, et al: Postoperative ischemic changes after glioma resection identified by diffusion-weighted magnetic resonance imaging and their association with intraoperative motor evoked potentials. Clinical article. J Neurosurg 119:829836,2013

16. Kamada K, Todo T, Ota T, Ino K, Masutani Y, Aoki S, et al: The motor-evoked potential threshold evaluated by tractography and electrical stimulation. Clinical article. J Neurosurg 111:785-795, 2009

17. Karnofsky DA Burchenal JH: The clinical evaluation of chemotherapeutic agents in cancer, in MacLeod CM (ed): Evaluation of Chemotherapeutic Agents. New York: Columbia University Press, 1949, p 196

18. Keles GE, Lundin DA, Lamborn KR, Chang EF, Ojemann G, Berger MS: Intraoperative subcortical stimulation mapping for hemispherical perirolandic gliomas located within or adjacent to the descending motor pathways: evaluation of morbidity and assessment of functional outcome in 294 patients. J Neurosurg 100:369-375, 2004

19. Kombos T, Süss O, Vajkoczy P: Subcortical mapping and monitoring during insular tumor surgery. Neurosurg Focus 27(4):E5, 2009

20. Kreth FW, Thon N, Simon M, Westphal M, Schackert G, Nikkhah G, et al: Gross total but not incomplete resection of glioblastoma prolongs survival in the era of radiochemotherapy. Ann Oncol 24:3117-3123, 2013

21. Lacroix M, Abi-Said D, Fourney DR, Gokaslan ZL, Shi W, DeMonte F, et al: A multivariate analysis of 416 patients with glioblastoma multiforme: prognosis, extent of resection, and survival. J Neurosurg 95:190-198, 2001

22. Maesawa S, Fujii M, Nakahara N, Watanabe T, Wakabayashi $\mathrm{T}$, Yoshida J: Intraoperative tractography and motor evoked potential (MEP) monitoring in surgery for gliomas around the corticospinal tract. World Neurosurg 74:153-161, 2010

23. McGirt MJ, Chaichana KL, Attenello FJ, Weingart JD, Than K, Burger PC, et al: Extent of surgical resection is independently associated with survival in patients with hemispheric infiltrating low-grade gliomas. Neurosurgery 63:700-708, 2008

24. Medical Research Council: Aids to the Examination of the Peripheral Nervous System, Memorandum no. 45. London: Her Majesty's Stationery Office, 1981

25. Mikuni N, Okada T, Nishida N, Taki J, Enatsu R, Ikeda A, et al: Comparison between motor evoked potential recording and fiber tracking for estimating pyramidal tracts near brain tumors. J Neurosurg 106:128-133, 2007

26. Neuloh G, Pechstein U, Schramm J: Motor tract monitoring during insular glioma surgery. J Neurosurg 106:582-592, 2007

27. Nossek E, Korn A, Shahar T, Kanner AA, Yaffe H, Marcovici $\mathrm{D}$, et al: Intraoperative mapping and monitoring of the corticospinal tracts with neurophysiological assessment and 3-dimensional ultrasonography-based navigation. Clinical article. J Neurosurg 114:738-746, 2011

28. Ohue S, Kohno S, Inoue A, Yamashita D, Harada H, Kumon Y, et al: Accuracy of diffusion tensor magnetic resonance imaging-based tractography for surgery of gliomas near the pyramidal tract: a significant correlation between subcortical electrical stimulation and postoperative tractography. Neurosurgery 70:283-294, 2012

29. Penfield W, Boldrey E: Somatic motor and sensory representation in the cerebral cortex of man as studied by electrical stimulation. Brain 60:389-443, 1937

30. Prabhu SS, Gasco J, Tummala S, Weinberg JS, Rao G: Intra- 
operative magnetic resonance imaging-guided tractography with integrated monopolar subcortical functional mapping for resection of brain tumors. Clinical article. J Neurosurg 114:719-726, 2011

31. Raabe A, Beck J, Schucht P, Seidel K: Continuous dynamic mapping of the corticospinal tract during surgery of motor eloquent brain tumors: evaluation of a new method. Clinical article. J Neurosurg 120:1015-1024, 2014

32. Roberts DW, Valdés PA, Harris BT, Fontaine KM, Hartov A, Fan X, et al: Coregistered fluorescence-enhanced tumor resection of malignant glioma: relationships between $\delta$-aminolevulinic acid-induced protoporphyrin IX fluorescence, magnetic resonance imaging enhancement, and neuropathological parameters. Clinical article. J Neurosurg 114:595-603, 2011

33. Sala F, Lanteri P: Brain surgery in motor areas: the invaluable assistance of intraoperative neurophysiological monitoring. J Neurosurg Sci 47:79-88, 2003

34. Sanai N, Polley MY, McDermott MW, Parsa AT, Berger MS: An extent of resection threshold for newly diagnosed glioblastomas. Clinical article. J Neurosurg 115:3-8, 2011

35. Schucht P, Beck J, Abu-Isa J, Andereggen L, Murek M, Seidel $\mathrm{K}$, et al: Gross total resection rates in contemporary glioblastoma surgery: results of an institutional protocol combining 5-aminolevulinic acid intraoperative fluorescence imaging and brain mapping. Neurosurgery 71:927-936, 2012

36. Schucht P, Knittel S, Slotboom J, Seidel K, Murek M, Jilch A, et al: 5-ALA complete resections go beyond MR contrast enhancement: shift corrected volumetric analysis of the extent of resection in surgery for glioblastoma. Acta Neurochir (Wien) 156:305-312, 2014

37. Seidel K, Beck J, Stieglitz L, Schucht P, Raabe A: Low-threshold monopolar motor mapping for resection of primary motor cortex tumors. Neurosurgery 71 (1 Suppl Operative): 104-115, 2012

38. Seidel K, Beck J, Stieglitz L, Schucht P, Raabe A: The warning-sign hierarchy between quantitative subcortical motor mapping and continuous motor evoked potential monitoring during resection of supratentorial brain tumors. Clinical article. J Neurosurg 118:287-296, 2013

39. Senft C, Bink A, Franz K, Vatter H, Gasser T, Seifert V: Intraoperative MRI guidance and extent of resection in glioma surgery: a randomised, controlled trial. Lancet Oncol 12: 997-1003, 2011

40. Stummer W, Novotny A, Stepp H, Goetz C, Bise K, Reulen HJ: Fluorescence-guided resection of glioblastoma multiforme by using 5-aminolevulinic acid-induced porphyrins: a prospective study in 52 consecutive patients. J Neurosurg 93: 1003-1013, 2000

41. Stummer W, Pichlmeier U, Meinel T, Wiestler OD, Zanella F, Reulen HJ: Fluorescence-guided surgery with 5-aminolevulinic acid for resection of malignant glioma: a randomised controlled multicentre phase III trial. Lancet Oncol 7:392-401, 2006

42. Stummer W, Reulen HJ, Meinel T, Pichlmeier U, Schumacher W, Tonn JC, et al: Extent of resection and survival in glioblastoma multiforme: identification of and adjustment for bias. Neurosurgery 62:564-576, 2008
43. Stummer W, Tonn JC, Mehdorn HM, Nestler U, Franz K, Goetz $\mathrm{C}$, et al: Counterbalancing risks and gains from extended resections in malignant glioma surgery: a supplemental analysis from the randomized 5-aminolevulinic acid glioma resection study. Clinical article. J Neurosurg 114:613-623, 2011

44. Stummer W, van den Bent MJ, Westphal M: Cytoreductive surgery of glioblastoma as the key to successful adjuvant therapies: new arguments in an old discussion. Acta Neurochir (Wien) 153:1211-1218, 2011

45. Stupp R, Mason WP, van den Bent MJ, Weller M, Fisher B, Taphoorn $\mathrm{MJ}$, et al: Radiotherapy plus concomitant and adjuvant temozolomide for glioblastoma. N Engl J Med 352:987-996, 2005

46. Szelényi A, Langer D, Beck J, Raabe A, Flamm ES, Seifert V, et al: Transcranial and direct cortical stimulation for motor evoked potential monitoring in intracerebral aneurysm surgery. Neurophysiol Clin 37:391-398, 2007

47. Szelényi A, Senft C, Jardan M, Forster MT, Franz K, Seifert V, et al: Intra-operative subcortical electrical stimulation: a comparison of two methods. Clin Neurophysiol 122:1470-1475, 2011

48. Taniguchi M, Cedzich C, Schramm J: Modification of cortical stimulation for motor evoked potentials under general anesthesia: technical description. Neurosurgery 32:219-226, 1993

49. Vecht CJ, Avezaat CJ, van Putten WL, Eijkenboom WM, Stefanko SZ: The influence of the extent of surgery on the neurological function and survival in malignant glioma. A retrospective analysis in 243 patients. J Neurol Neurosurg Psychiatry 53:466-471, 1990

50. Vogelbaum MA, Jost S, Aghi MK, Heimberger AB, Sampson JH, Wen PY, et al: Application of novel response/progression measures for surgically delivered therapies for gliomas: Response Assessment in Neuro-Oncology (RANO) Working Group. Neurosurgery 70:234-244, 2012

51. Weller M, van den Bent M, Hopkins K, Tonn JC, Stupp R, Falini A, et al: EANO guideline for the diagnosis and treatment of anaplastic gliomas and glioblastoma. Lancet Oncol 15:e395-e403, 2014

52. Zhu FP, Wu JS, Song YY, Yao CJ, Zhuang DX, Xu G, et al: Clinical application of motor pathway mapping using diffusion tensor imaging tractography and intraoperative direct subcortical stimulation in cerebral glioma surgery: a prospective cohort study. Neurosurgery 71:1170-1184, 2012

Manuscript submitted August 18, 2014.

Accepted October 7, 2014.

A proof-of-concept analysis, which included approximately onethird of the present patient population, was previously published (Raabe et al: J Neurosurg 120:1015-1024, 2014).

Please include this information when citing this paper: DOI: 10.3171/2014.10.FOCUS14524.

Address correspondence to: Philippe Schucht, M.D., Department of Neurosurgery, Bern University Hospital, Freiburgstrasse 10, 3010 Bern, Switzerland. email: philippe.schucht@insel.ch. 